\title{
e-Phaïstos
}

e-Phaïstos

Revue d'histoire des techniques / Journal of the history

of technology

IX-1 | 2021

Autour de Léonard de Vinci

\section{Les trois racines de la statique graphique}

De Léonard de Vinci à Karl Culmann

The Three Roots of Graphic Statics

\section{Martin Pospíšil}

\section{(2) OpenEdition}

\section{Journals}

Édition électronique

URL : https://journals.openedition.org/ephaistos/9020

DOI : $10.4000 /$ ephaistos. 9020

ISSN : 2552-0741

Éditeur

IHMC - Institut d'histoire moderne et contemporaine (UMR 8066)

Référence électronique

Martin Pospíšil, «Les trois racines de la statique graphique », e-Phaïstos [En ligne], IX-1 | 2021, mis en ligne le 27 avril 2021, consulté le 18 septembre 2021. URL : http://journals.openedition.org/ephaistos/ 9020 ; DOI : https://doi.org/10.4000/ephaistos.9020

Ce document a été généré automatiquement le 18 septembre 2021.

Tous droits réservés 


\title{
Les trois racines de la statique graphique
}

\author{
De Léonard de Vinci à Karl Culmann \\ The Three Roots of Graphic Statics
}

Martin Pospíšil

Dès son origine, la mécanique de construction a fait partie de l'architecture. Malgré de nombreuses difficultés, elle accompagne son évolution jusqu'à nos jours, en demeurant l'un de ses principaux piliers. Ses méthodes ressemblent parfois à la création artistique (Picon 1997); néanmoins, elle constitue un domaine extrêmement technique. Elle s'appuie de surcroît, sur les sciences fondamentales, physique, mathématiques, et géométrie. Depuis toujours étroitement entremêlée à la mécanique de construction, cette dernière contribua à son enrichissement par des moyens assez divers, depuis les règles de proportion de l'Antiquité et les compositions basées sur la construction du nombre d'or (Pospísil 2007), jusqu'à la statique graphique du XIX ${ }^{\mathrm{e}}$ siècle, en passant par les règles médiévales de géométrie et par la mécanique moderne révolutionnaire de Galilée (Culmann 1866, Benvenuto 1991a et b). Désormais, la majeure partie des spécialistes en statique utilisent l'informatique, mais sans lui faire totalement confiance : ils sont assez nombreux à vérifier leur calcul en utilisant cette méthode, désormais classique, élaborée au XIX ${ }^{\mathrm{e}}$ siècle : la statique graphique ${ }^{1}$.

2 La statique graphique est une méthode de « calcul par le trait » (Cousinéry 1839 :32), un "art of calculation by drawing lines" (Smith 1889:347) dont l'origine historique est fondée sur la mécanique et la géométrie. Cette méthode géométrique de calcul destinée à appréhender visuellement et à résoudre concrètement des problèmes de la mécanique (newtonienne) des solides, a évolué sous l'influence des pratiques de construction (Pospísil 2016). Après des siècles d'évolution autonome, toutes ces sources se sont interpénétrées pour créer, grâce à certains excellents mathématiciens, géomètres et ingénieurs, une ingénieuse méthode de calcul. Il revint à l'ingénieur allemand Karl Culmann², qui enseignait le Génie civil à Zurich, d'en assurer la formulation complète au milieu des années 1860 (Culmann 1966). Ses travaux ont été largement utilisés jusqu’à la Première Guerre mondiale. Les dessins de constructions 
des treillis de bâtiments célèbres, tels à Prague, le Rudolfinum, le Théâtre national ou bien la cathédrale de Saint Guy, Saint Venceslas et Saint Adalbert, tous achevés à cette époque, témoignent du succès de la méthode auprès des praticiens. Mais le facteur principal de l'évolution de la statique graphique fut le développement des voies de chemin de fer et des routes partout en Europe.

Bien que la statique graphique ait été remplacée, petit à petit, par des méthodes analytiques et numériques plus universelles et plus précises (Chobot et al., 1982), elle est restée, en pratique, une méthode de vérification privilégiée auprès de nombreux experts en statique ${ }^{3}$, y compris pour les plus grandes constructions contemporaines. L'avantage de cette méthode est sa concision. Elle se signale aussi, du fait de la forme graphique du calcul, par sa clarté, son caractère démonstratif, à quoi s'ajoute l'impossibilité de commettre, en principe, une erreur de calcul majeure. Par contre, elle a pour inconvénient, une précision moindre et la possibilité limitée de s'adapter à des constructions toujours plus complexes. Il y a plusieurs années, cette technique a été reprise au MIT (Massachusetts Institute of Technology) dans la formation d'architectes et de spécialistes des domaines autres que l'ingénierie, d'où elle a été reprise par d'autres écoles et pays. Actuellement, la statique graphique connaît une renaissance remarquable en particulier aux États-Unis et dans d'autres pays d'Europe occidentale, même si elle est utilisée dans des disciplines principalement non techniques, en médecine par exemple, pour interpréter les mécanismes du mouvement du corps humain. Et des recherches détaillées sur de nouvelles méthodes de calcul basées sur la statique graphique, combinées avec des méthodes probabilistes sont en cours, à l'Eidgenössische Technische Hochschule (ETH) de Zurich par exemple, sous l'égide du professeur P. Block, ancien élève du professeur Ochsendorf au MIT. Enfin, un nombre considérable de manuels et de textes, y compris des réimpressions historiques, sont actuellement publiés.

Les débuts de la statique graphique sont généralement associés à la publication de l'ouvrage en huit volumes de Karl Culmann, Die Graphische Statik, entre 1864 et 1866, à Zurich, en Suisse. Toutefois, comme Culmann le souligne lui-même dans l'introduction de son travail, il s'inscrit dans les pas de plusieurs de ses contemporains qui ont préparé la voie quelques années avant lui. Il cite ainsi dans sa préface, Jean-Victor Poncelet (1788-1867), Barthélémy Édouard Cousinery (1790-1851), Jakob Steiner (1796-1863), Karl Georg Christian Staudt (1798-1867) et quelques autres (Culmann 1866 :V-XVI). Mais, pour lui, la statique graphique plonge ses racines, bien en amont, dans trois domaines: le dessin technique ("das Zeichen ist seine Sprache», Culmann $1866: \mathrm{V})$, la géométrie projective (Culmann $1866: \mathrm{VI})$ et bien sûr, la mécanique statique. Prolongeant cette argumentation, nous observerons, dans un premier temps, les trois courants élémentaires sur lesquels la statique graphique s'est fondée et dont elle s'est inspirée, avant de les réunir, à savoir : i) la composition et la décomposition des forces et du polygone de forces fermé ; ii) l'impulsion donnée par les pratiques, autrement dit, le besoin de procédures de construction en tant que force motrice de base; iii) la dualité forme-force et l'utilisation d'un polygone funiculaire pour calculer une construction réelle. Dans un deuxième temps, nous évoquerons l'évolution de la statique graphique dans le dernier tiers du XIX ${ }^{\mathrm{e}}$ siècle. 


\section{Composition et décomposition des forces : les débuts du polygone des forces fermé}

\section{Léonard de Vinci}

Outre les connaissances de base sur la force dans la physique des temps anciens et du Moyen Âge ${ }^{4}$, nous pouvons trouver des indices concrets sur la manière de résoudre la composition et la décomposition des forces dans les ouvrages de Léonard de Vinci tels que le Codex de Madrid ${ }^{5}$ ou encore le Codex d'Arundel ${ }^{6}$. Léonard de Vinci examine la décomposition des forces dans plusieurs énoncés pratiques, par exemple la conception de l'arbalète ${ }^{7}$ ou du système de poulies ${ }^{8}$, ainsi que pour illustrer ses réflexions théoriques, comme dans l'étude de la charge remorquée ${ }^{9}$ ou de la déformation d'une canne flexible ${ }^{10}$. La répartition des efforts sur deux câbles suspendus y est clairement démontrée (fig.1) ${ }^{11}$.

Fig.1. Léonard de Vinci : Décomposition des forces
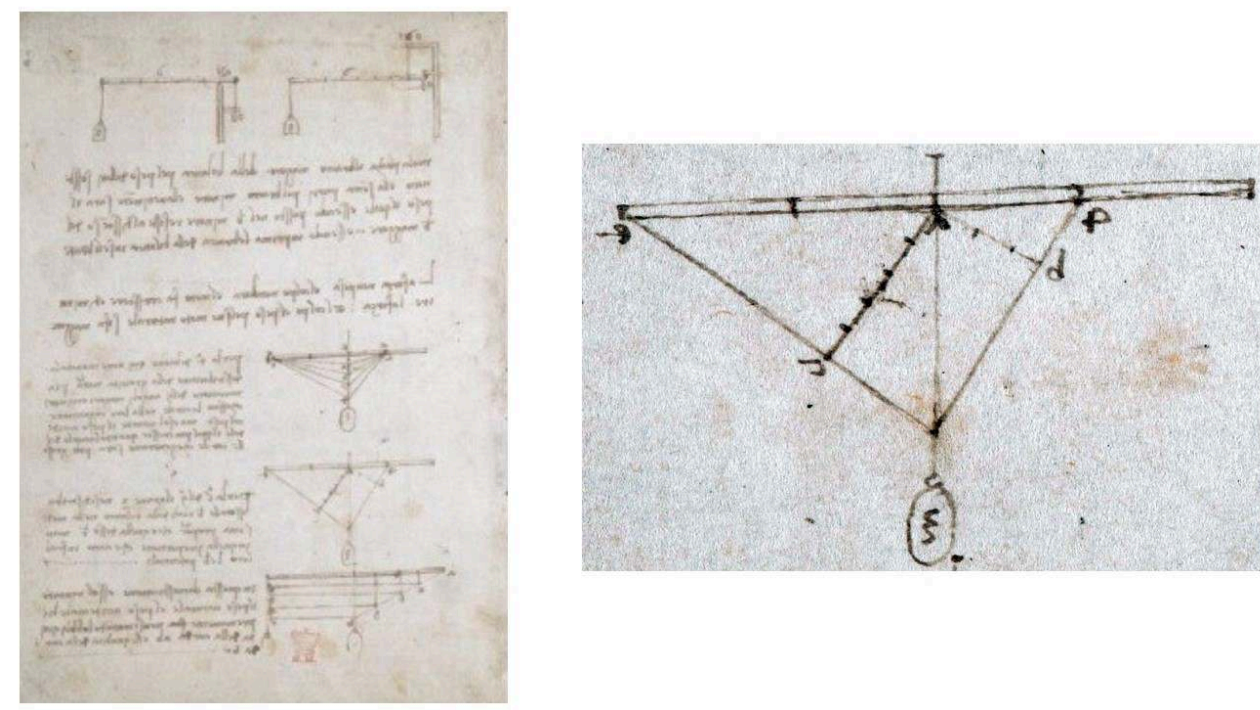

Depuis la canne horizontale de départ, deux cordes asymétriques sont suspendues suivant son axe vertical (deux parties à gauche pour la corde gauche et une partie à droite pour la corde droite). Elles sont reliées l'une à l'autre sur l'axe vertical de la canne. Au niveau de ce point, un poids est suspendu. Source : Léonard de Vinci, Codex Arundel, 1478-1518, British Museum, Londres, MS 263, fol.1 verso, détails

$6 \quad$ Le principe du calcul de Léonard de Vinci repose sur un équilibre momentané : les cordes représentent les porteurs de deux forces inconnues qui agissent sur deux bras différents émergeant du point commun, le centre de rotation. Le centre de rotation correspond au centre de la canne et les bras de force constituent des distances perpendiculaires entre le centre de la canne et les cordes.

Léonard de Vinci était déjà très proche du vrai parallélépipède de forces. Si, au lieu de présenter une position générale, les cordes formaient un angle droit, nous serions en présence d'un vrai parallélépipède de forces, même si dans ce cas particulier, il ne s'agit que d'orthogonalité de la force. Léonard n'a donc pas résolu le problème graphiquement mais numériquement, en se fondant sur les conditions momentanées d'équilibre (Pisano, Capecchi 2016). Cela étant, les connaissances de Léonard de Vinci 
dans ce domaine n'ont pas retenu immédiatement l'attention. Elles sont demeurées plus ou moins inaperçues jusqu'en 1797 (Venturi 1797) ${ }^{12}$.

\section{Simon Stevin et Galilée}

Le mathématicien et physicien flamand Simon Stevin ${ }^{13}$, autre excellent penseur de la Renaissance, s'est intéressé à l'équilibre et la décomposition des forces. Il parvint à apporter une preuve de la validité de la loi de l'équilibre des forces sur un plan incliné, phénomène qu'il décrit dans son livre De Beghinselen der weeghconst ${ }^{14}$. Il s'agit d'une expérience physique à la fois sophistiquée et très simple. Elle est réalisée avec une chaine composée de sphères de taille et poids égaux, suspendues à deux plans adjacents et différemment inclinés ${ }^{15}$ (fig.2).

Fig.2. Simon Stevin : Preuve de la validité de la loi d'équilibre sur un plan incliné
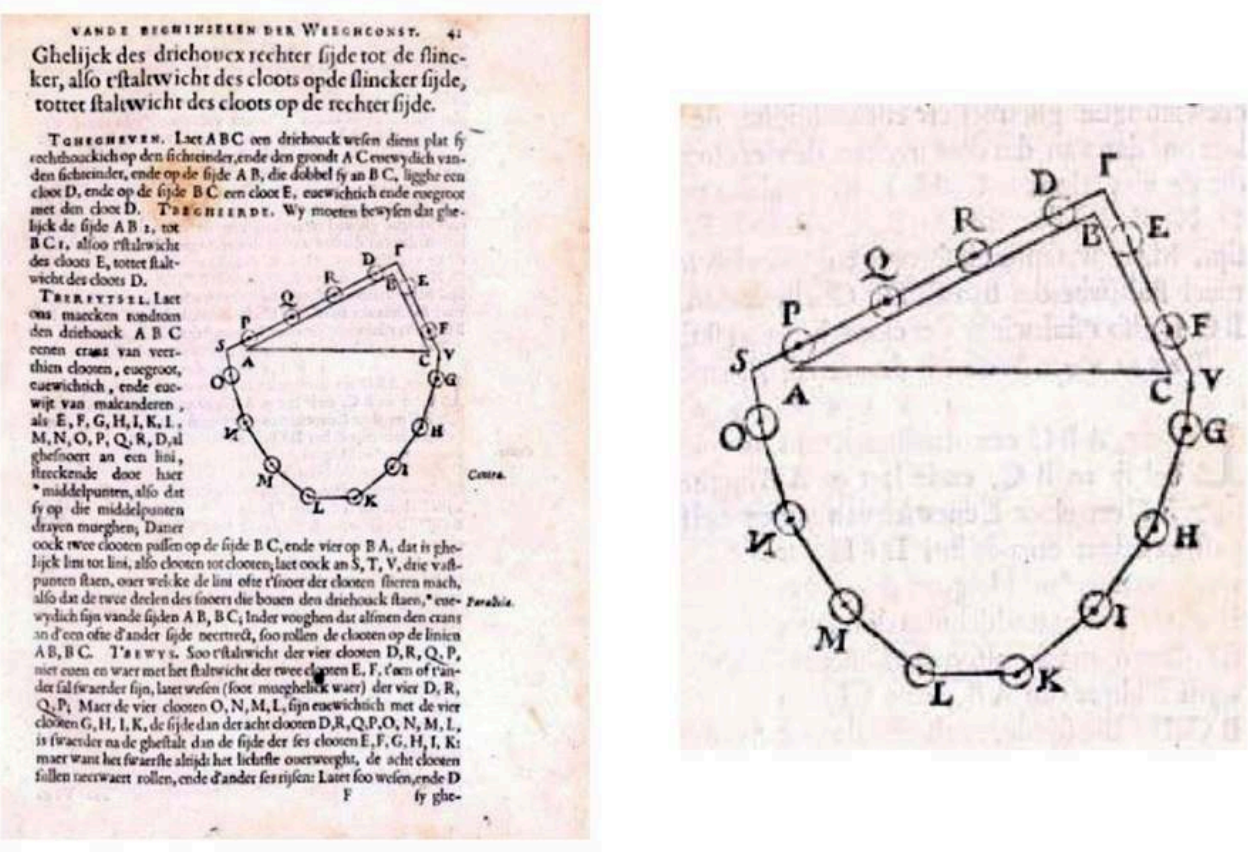

À partir des recherches de Stevin, mais aussi de notre propre expérience, la chaîne enroulée autour du prisme est stable dans n'importe quelle position. Si la partie inférieure de la chaîne est déconnectée, la partie supérieure de la chaîne reste stable ; elle ne glissera ni à droite ni à gauche, car le fond déconnecté étant idéalement symétrique, sollicite les deux côtés de la partie supérieure de la même manière. Cela signifie que les composantes verticale et horizontale des forces de charge uniformes sur les deux côtés du plan incliné sont égales. Elles sont aussi égales aux composantes verticale et horizontale des réactions respectives.

Source : Simon Stevin (Stevins dans l'épitaphe), De Beghinselen der weeghconst, Leyden, 1586, p.41, détail

9 Par son expérience, Stevin a donc créé le premier polygone des forces fermé (Benvenuto 1991). Comme on peut le voir dans la série de gravures de la deuxième partie du livre, Stevin s'est également attaché à une représentation graphique de la décomposition des forces, grâce à laquelle il s'est rapproché encore plus du parallélépipède de Léonard de Vinci. En mesurant la force nécessaire pour déplacer la charge sur un plan incliné, il a raisonné avec une composante gravitationnelle et une autre agissant perpendiculairement à la surface en dessous. En fonction de l'angle du plan incliné, ces dernières soit se chevauchent (la force nécessaire pour maintenir la 
charge au repos étant égale à zéro) soit elles dévient progressivement jusqu'à la position théoriquement verticale du plan incliné (la force nécessaire pour maintenir la charge au repos étant exactement égale à la masse de la charge). À en juger par le choix des charges (wagon et bateau), il est évident que Stevin était conscient du problème du frottement entre la charge et la surface en dessous.

Un peu plus jeune que Stevin, Galilée (1564-1642), ingénieur, savant et philosophe, peut quant à lui être considéré comme le fondateur de la mécanique moderne ${ }^{16}$. Il a consacré au raisonnement concernant la décomposition des forces une partie du Quatrième discours (le Quatrième jour), des Discorsi, son œuvre capitale sur les mathématiques et la mécanique $^{17}$. Dans ce dialogue, il aborde la question de la balistique, à travers le mouvement d'un projectile. Tiré à une certaine vitesse, le projectile subit au cours de son trajet les effets de la gravité terrestre. Galilée a fait insérer dans le texte, avant les tableaux balistiques finaux, une branche du mouvement du corps sur différents plans inclinés ${ }^{18}$. Il a mesuré la force exercée par le corps dans la direction du plan incliné de la même manière que Stevin, sur la poulie avec le poids. Galilée a également constaté que cette force augmentait en accroissant l'inclinaison. Cependant, il n'est pas parvenu à approfondir la question du parallélogramme des forces.

\section{Isaac Newton et Pierre Varignon}

11 Isaac Newton a abordé la problématique de la décomposition des forces d'une manière tout à fait différente ${ }^{19}$. Dans son œuvre principale, Principes mathématiques de la philosophie naturelle ${ }^{20}$, il utilisait déjà le parallélogramme des forces sous la forme que nous connaissons encore. Au sein du chapitre qui définit ses fameuses lois du mouvement (Axiomata sive Leges Motus), plus précisément dans le Corollaire $\mathrm{n}^{\circ} 1$ (Corol.I), il décrit de manière très exacte la construction d'un parallélogramme des forces: "Lorsque deux forces sont combinées, leur somme sera la diagonale d'un parallélépipède, où ces forces sont les côtés » (fig.3). 
Fig.3. Isaac Newton : Description exacte de l'effort parallèle

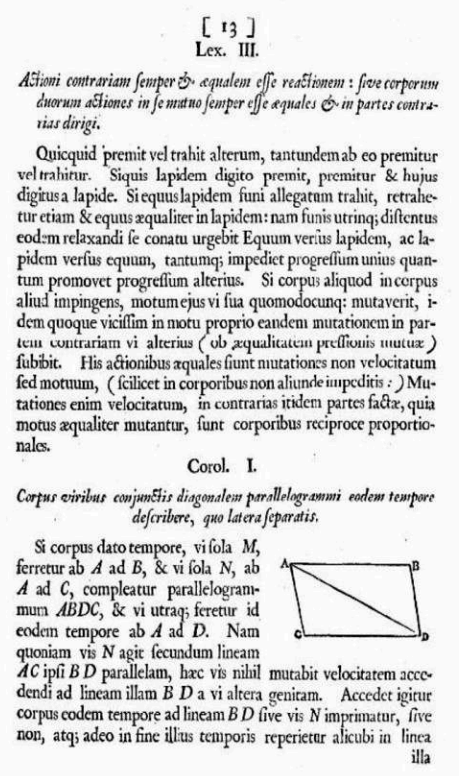

Corol. I.

Corpus viribus conjunctis diagonalem parallelogrammi eodem tempore defcribere, quo latera feparatis.

Si corpus dato tempore, vi fola $M$, ferretur ab $A$ ad $B$, \& vi fola $N$, ab

$A$ ad $C$, compleatur parallelogrammum $A B D C$, \& vi utraq; feretur id codem tempore $\mathrm{ab} A$ ad $D$. Nam quoniam vis $N$ agit fecundum lineam

$A C$ ipf $B D$ partelam, hac vis dendi ad lineam illam $B D$ a vi altera genitam. Accedet igitur corpus eodem tempore ad lineam $B D$ five vis $N$ imprimatur, five non, atq; adeo in fine illius temporis reperietur alicubi in linea

Corol.I. « Corpus viribus conjunctis diagonalem parallelogrammi eodem tempore describere, quo latera separatis » : «Lorsque deux forces sont combinées, leur somme sera la diagonale d'un parallélépipède, où ces forces sont les côtés».

Source : Philosophiæ Naturalis Principia Mathematica, Londres, 1687, vol. Axiomata sive Leges Motus, p. 13

Pour Newton, le parallélogramme des forces constituait l'expression de l'interaction de deux forces conduisant à l'accélération ou au ralentissement du corps, décrivant ainsi non pas une situation statique, mais dynamique. Bien que sa définition de la somme des forces, illustrée par le dessin et la description mathématique exacte, soit très simple et même élémentaire, il a fallu encore deux siècles avant qu'elle ne soit reprise par la statique; quant à la discussion sur la preuve mathématique de la véracité du parallélogramme des forces, elle s'est prolongée jusqu'au début du $\mathrm{XX}^{\mathrm{e}}$ siècle (Lange 2009).

Isaac Newton correspondait avec le mathématicien français Pierre Varignon (1654-1722) ${ }^{21}$. Cet ami, un peu plus âgé que lui, avait publié en 1687, la même année donc que les Principes, son ouvrage majeur dans le domaine de la mécanique des structures, Nouvelle mécanique ou Statique ${ }^{22}$. Varignon, de la même manière que Léonard de Vinci, a recouru, pour sa démonstration, à des expériences reposant sur un système de cordes. Cependant, contrairement à Léonard, ces cordes sont clairement porteuses des forces (fig.4). De plus, il forme progressivement un polygone en corde à partir du système de forces et, dans le même esprit que Stevin, il le ferme in fine, formant ainsi un polygone des forces correspondant (fig.5 ; voir également Chatzis 2004, Kurrer 2008, Bénard 2009). 
Fig.4. Pierre Varignon : les cordes comme porteurs de forces
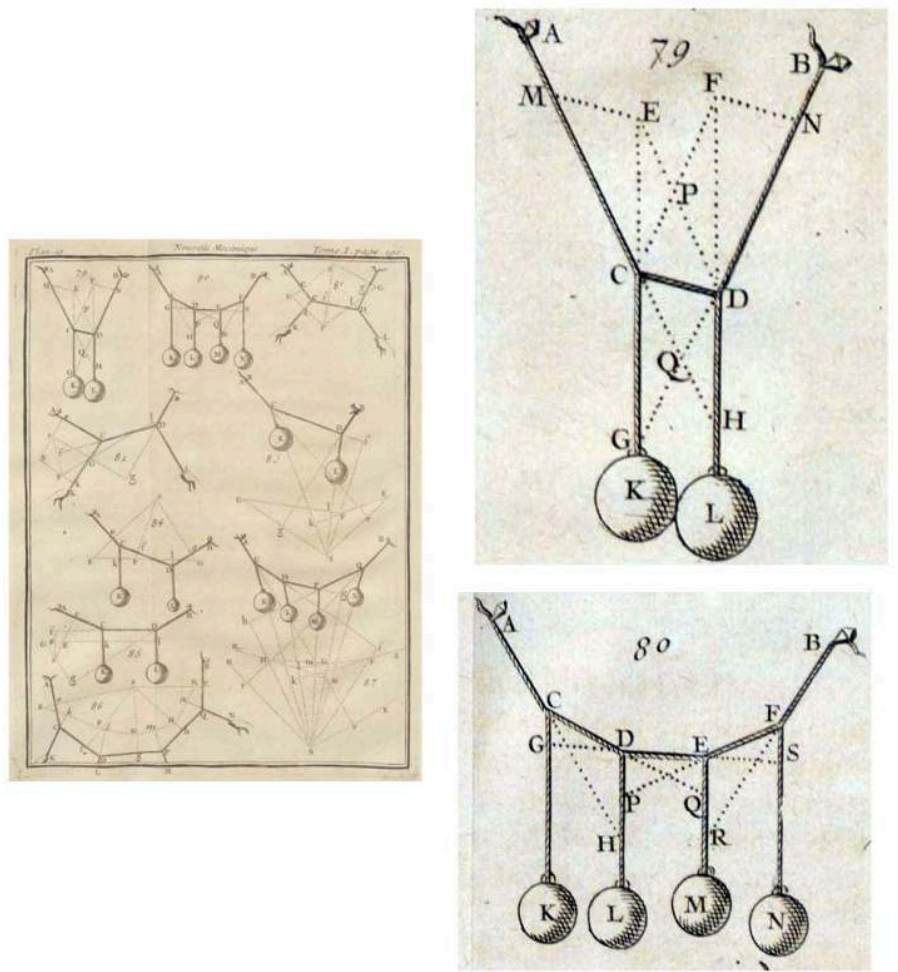

Source : Varignon, Nouvelle mécanique ou Statique, Paris, 1687, Tome Ier, p.190

Fig.5. Pierre Varignon : Fermeture d'un polygone de force
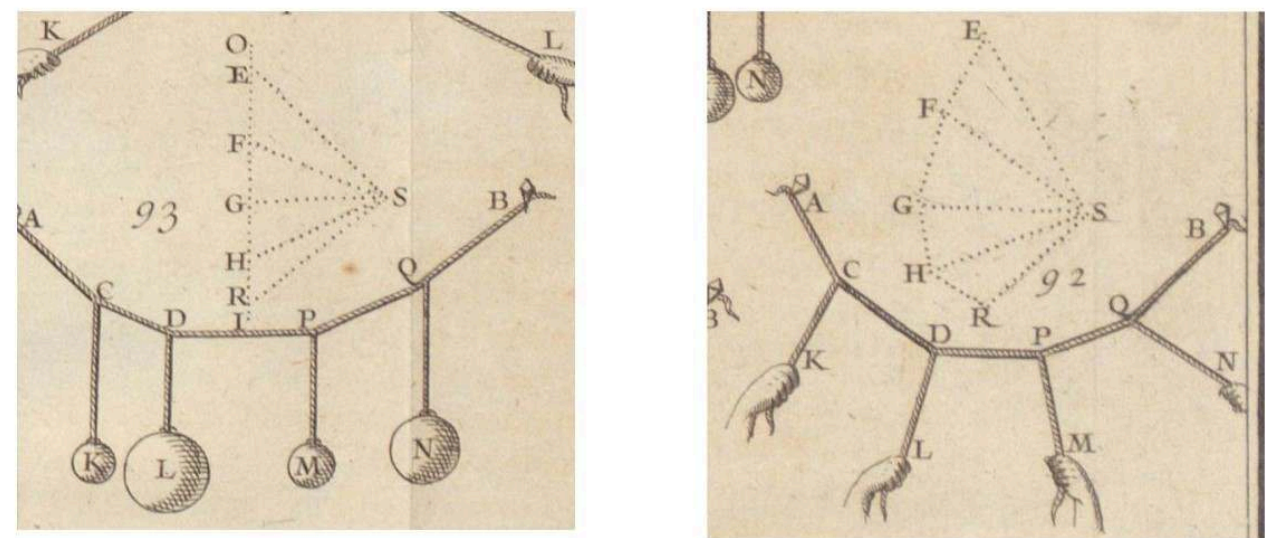

Dans la découpe en bas à gauche, nous voyons une corde inflexible attachée entre les points $A$ et $B$ avec des poids suspendus $K, L, M, N$. La position d'équilibre résultante des cordes $A, C, D, P, Q, B$ est appelée le polygone funiculaire. Sur la même figure, on peut voir le polygone des forces $S, E, F, G, H, R$, I, qui est une suite de triangles en équilibre, ainsi le triangle $S, E, F$ exprime l'équilibre au nœud $C$.

Source : Varignon, Nouvelle mécanique ou Statique, Paris, 1687, Tome Ier, p. 210

Enfin, de la même manière que Léonard de Vinci, Varignon travaille avec les bras de levier. Il formule ce que l'on appelle le théorème du moment selon lequel le moment résultant est égal au moment des composantes individuelles (fig.6). 

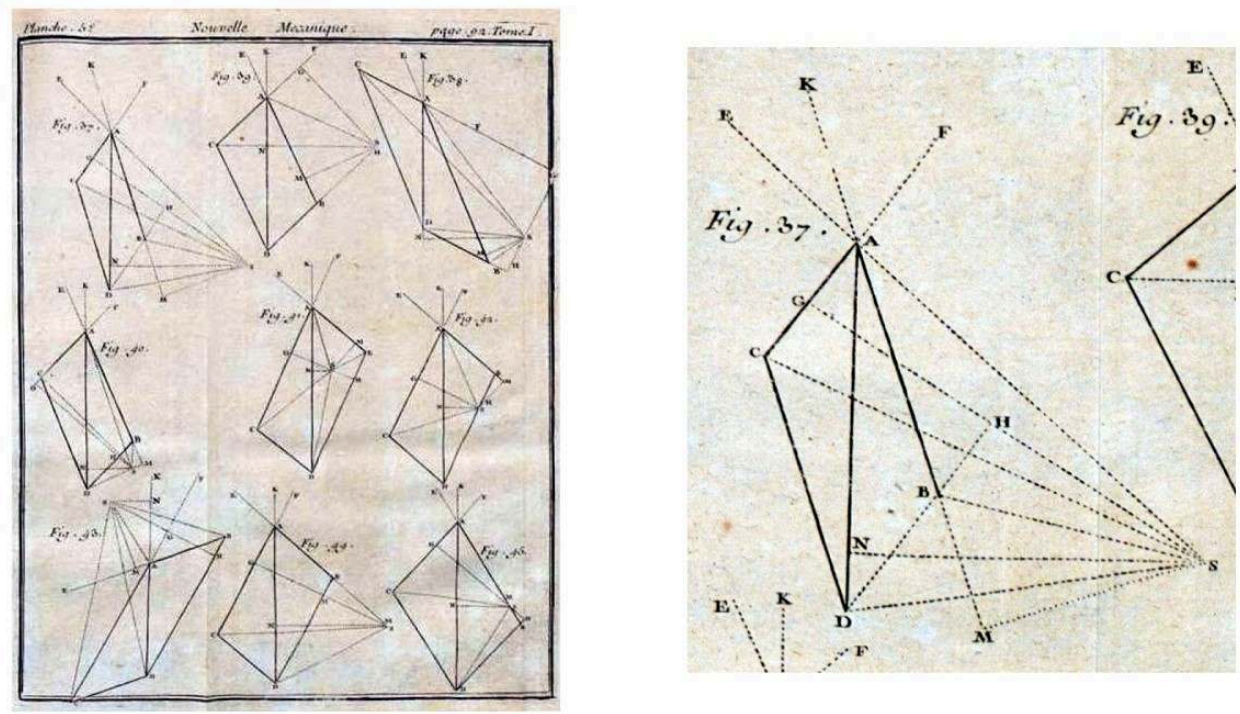

«Le parallélogramme A B C D montre les sections déterminantes des composants, sa diagonale AD est la section déterminante de la résultante. Les points $G, H, M, N$ sont les talons des perpendiculaires abaissées du centre de moment $S$ aux côtés $A C, B D, A B, A D$. Selon le théorème de Varignon, le moment de la résultante est égal aux sommes du moment des composantes. Par conséquent, $A D$ s'applique. NS = AB. SM + AC. GS ... ».

Source : Varignon, Nouvelle mécanique ou Statique, Paris, 1687, Tome |er, p. 92

Dans la deuxième partie de son livre, Varignon aborde la solution vectorielle d'un système à trois forces, l'une des méthodes graphiques de base utilisées pour résoudre le système de forces, par exemple lors de l'ancrage des mâts au moyen d'un triplet de cordes. Il préfigurait ainsi l'utilisation directe de la méthode graphique pour les calculs en mécanique du bâtiment.

\section{Voûtes, arcs et voussoirs : l'impulsion donnée par les applications pratiques}

Varignon fit des émules à l'Académie royale des Sciences. Peu après la publication de sa Nouvelle mécanique de Varignon, Philippe de la Hire (1640-1718 ${ }^{23}$, s'intéressa à l'application pratique du polygone funiculaire (voir définition en fig.5). La Hire avait publié en 1673 sa Nouvelle méthode en géométrie ${ }^{24}$, un ouvrage que Varignon maîtrisait parfaitement et qu'Isaac Newton connaissait sans doute aussi ${ }^{25}$. Pour autant, chacun d'eux a pensé la question de la décomposition des forces d'une manière différente. La Hire, dans son Traité de Mécanique (La Hire 1695) utilise le polygone des forces et le polygone funiculaire correspondant pour calculer les forces inconnues dans l'arc ${ }^{26}$. Il tablait alors sur un contact parfaitement glissant entre les voussoirs individuels, hypothèse qu'il modifia ensuite (La Hire 1712) pour considérer que la friction entre les voussoirs était trop importante pour qu'un mouvement puisse se produire entre eux. Mais le fait qu'ils soient en mesure de pivoter légèrement quand le support subit une déformation, le conduisit à s'interroger sur le comportement de l'arc et à proposer une solution ${ }^{27}$ qu'aucun savant ne parvint à égaler dans les décennies suivantes ${ }^{28}$, et qui contribua largement à la compréhension de la mécanique des arcs. De son côté, Charles Étienne Louis Camus (1699-1768) ${ }^{29}$, également disciple de Varignon, développa 
l'utilisation du polygone funiculaire pour déterminer la taille des forces de traction, notamment un polygone dimensionnel subissant les forces multidirectionnelles (Camus 1749-1752, fig.7 $)^{30}$.

Fig.7. Camus : le polygone funiculaire

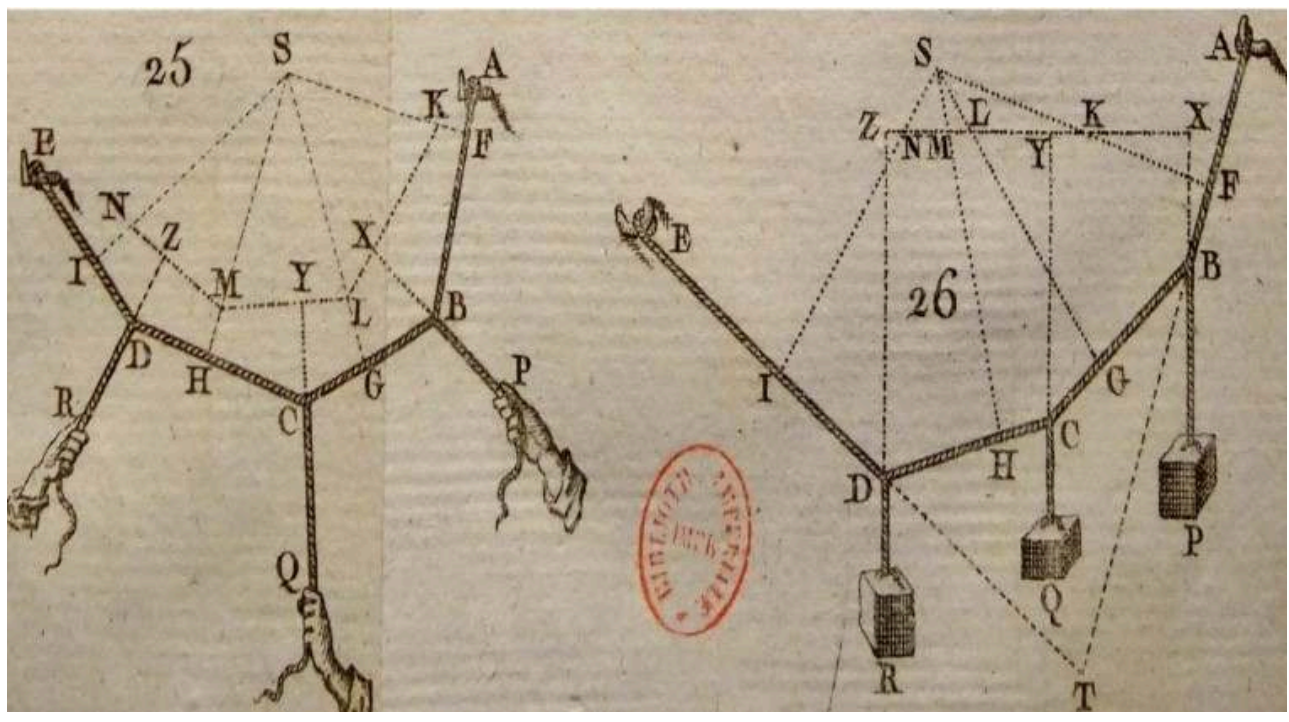

Source : Charles Étienne Louis Camus, Cours de mathématique, Paris 1749-52, Partie III, Tome II, titul.libri, pl.III, p.58, détail fig.25, fig.26

En Italie, le physicien et mathématicien italien Giovanni Poleni (1683-1761) ${ }^{31}$ développa une application très intéressante dont on peut considérer qu'elle constitue une étape décisive vers la statique graphique. À la charnière des XVII et $\mathrm{XVIII}^{\mathrm{e}}$ siècles, la voûte de la cathédrale du Vatican à Rome commença à se fissurer sérieusement. Tommaso Le Seur, François Jacquier et Ruggiero Giuseppe Boscovich, trois jésuites réputés pour leurs connaissances en mathématiques, furent appelés de France pour résoudre le problème posé par la restauration ${ }^{32}$. En 1742 , les «tre matematici" comme on prit l'habitude de les appeler, procédèrent au calcul de la capacité de charge de la voûte ${ }^{33}$. Leur solution consista à renforcer la voûte au moyen de cerceaux forgés (fig.8). 
Fig.8. Leseur, Jacquier, Boscovich : dessin des fissures et réflexion sur les forces en jeu dans la coupole de Saint-Pierre au Vatican
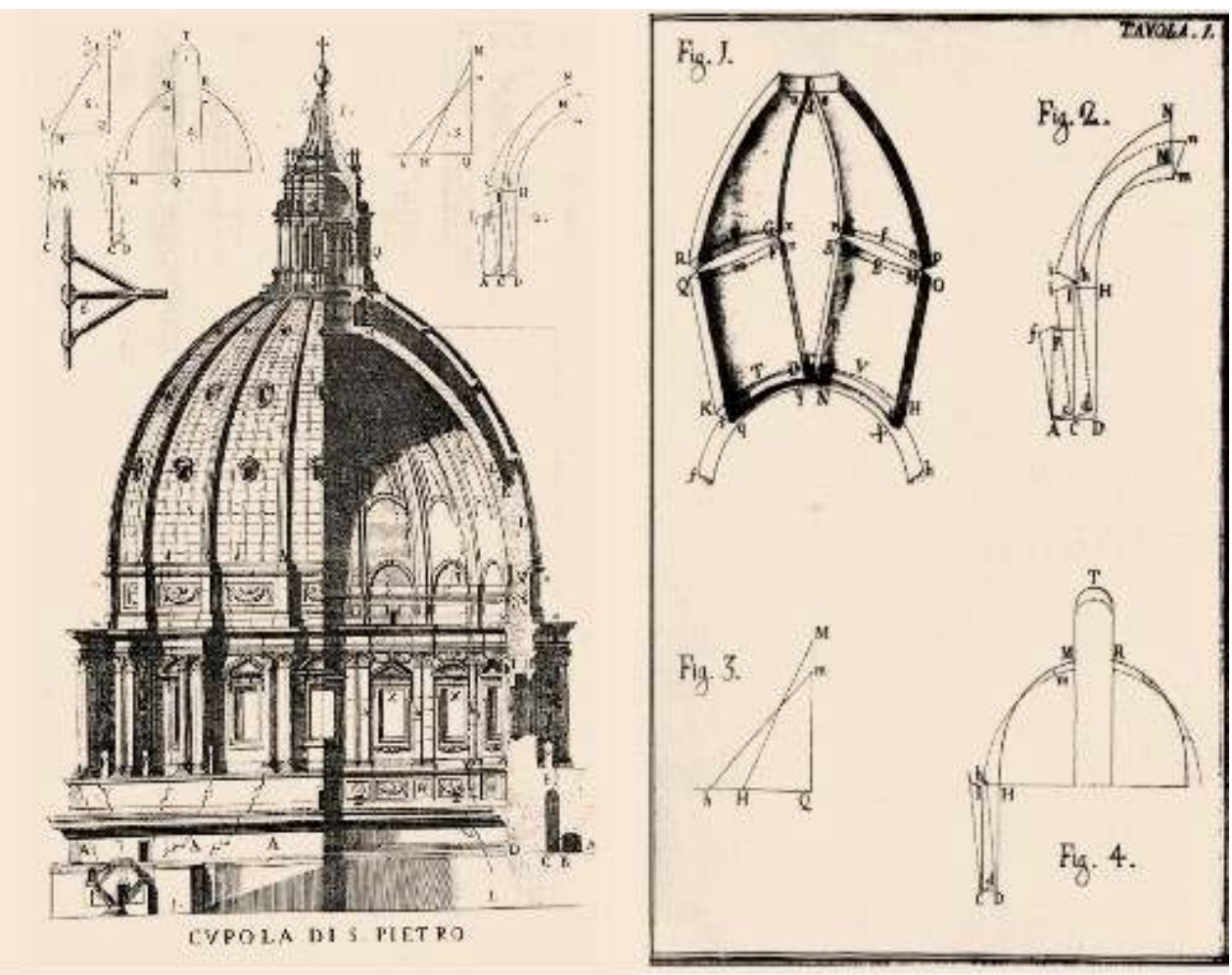

Source: Poleni's Manuscripts about the Dome of Saint Peter's (Lopez 2006 :1959 et 1961)

En complément, Poleni développa une évaluation de sécurité graphique de contrôle de la voûte en utilisant le principe de la chaînette renversée qu'avait découvert Robert Hooke, en 1675 (Lopez 2006) ${ }^{34}$. Le diagramme qu'il propose tend à prouver qu'il n'a pas seulement considéré la chaînette mathématique : on voit au dessin qu'il fournit dans son manuscrit, qu'il a travaillé sa forme au moyen de la taille graduée des sphères créant de facto une ligne de pression de la voûte; ensuite il a vérifié si la ligne ne dépassait pas la masse du dôme (fig.9). 
Fig.9. Giovanni Poleni : différence entre la forme réelle de la coupe du dôme et le cours de la chaîne inversée

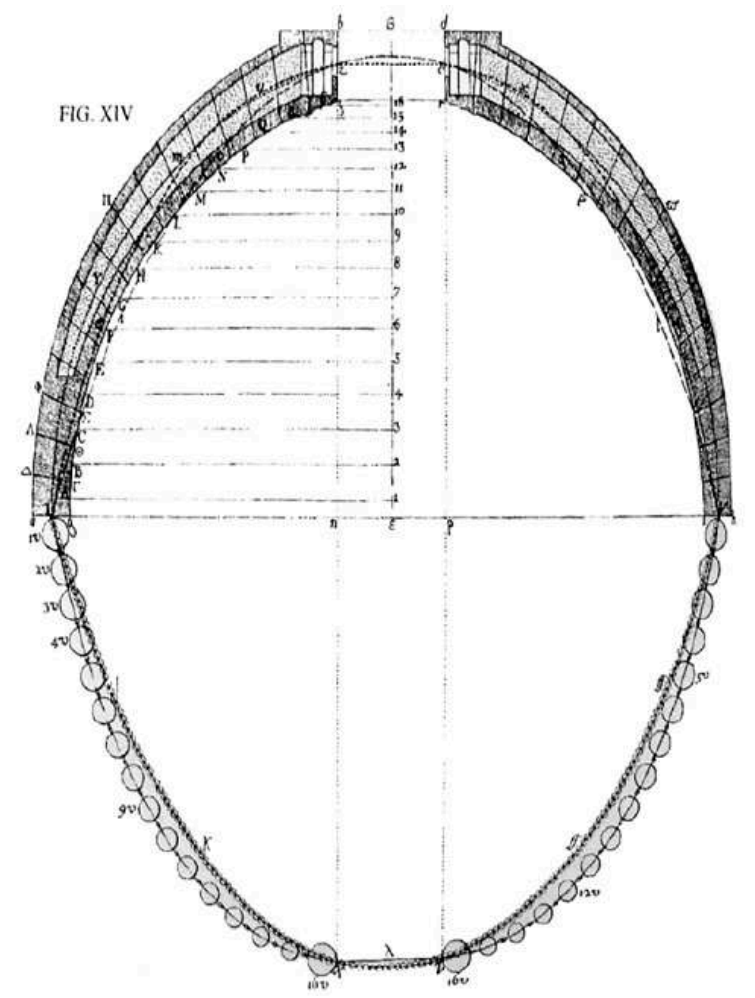

Source : Heyman 1998 (2003) : 86-90

Mentionnons enfin la stéréotomie, en tant qu'étape majeure et source d'inspiration pour la statique graphique. La stéréotomie s'apparente à la discipline qui résout les problèmes structurels des constructions (murs, voûtes, escaliers, traverses). Elle étudie comment former des corps à partir d'un matériau donné et comment les lier pour former la construction du projet ${ }^{35}$. Dans le traité de stéréotomie qu'il publie entre 1737 et 1739, Amédée François Frézier donne la primauté de l'usage de la notion au De Lapidum Sectione, le traité de coupe de pierres, publié en 1672 par le Père Deschalles, un jésuite, initialement professeur de navigation à l'Académie de marine à Marseille (Frézier $1737: \mathrm{XIII})^{36}$. Les liens entre la stéréotomie et la mécanique du bâtiment sont très étroits ${ }^{37}$. Frézier indique également que les premières tentatives documentées de formation d'articulations sur les voûtes, qui sont liées à la représentation graphique du cours des forces, sont dues à Augustin A. Danyzy qui dessina des modèles stéréotomiques de voûtes en $1732^{38}$. Développant l'idée de pivotement des voussoirs, il démontra leur possible effondrement (fig.10). 
Fig.10. Augustin A. Danyzy : recherche de l'effondrement de l'arc

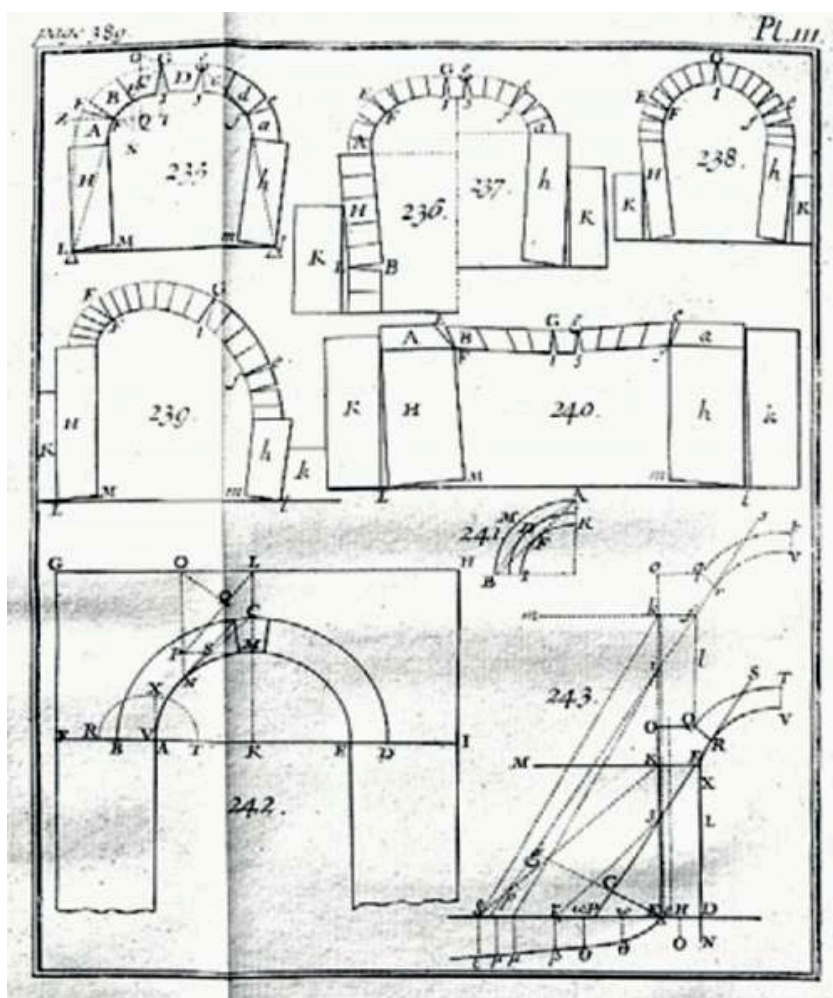

La recherche se fait en utilisant la stéréotomie pour essayer de trouver une solution graphique. Source : Frézier 1739, t.III, p.389, pl.111

\section{Lamé, Clapeyron, Poncelet et le calcul des constructions réelles : la dualité forme-force}

Les ingénieurs Benoît Clapeyron et Gabriel Lamé furent les premiers à utiliser le polygone funiculaire de Varignon (Kurrer 2008) ${ }^{39}$. Ils parvinrent à résoudre le cours des forces dans une construction réelle, en employant la graphique. Devenus membres du corps des Mines en 1820, ils partirent ensemble enseigner les mathématiques pures et appliquées à l'École des Travaux publics de Saint-Pétersbourg, une position qu'ils exercèrent jusqu'en 1830. La question de la construction du dôme de l'église SaintIsaac, à Saint-Pétersbourg étant en discussion depuis 1816 (Fedorov 2015), ils sentirent la nécessité de reprendre la théorie des voûtes, déjà traitée par Navier (Lapparent 1897). Ils calculèrent l'arc situé sous le tambour et ils adressèrent à l'Académie des sciences un mémoire sur ce sujet, qui mérita les louanges de Prony et fut publié dans les Annales des Mines en 1823 (Lamé, Clapeyron 1823) ${ }^{40}$. Ils utilisèrent la dualité géométrique entre le polygone funiculaire et des forces pour calculer le cours des forces de pression dans l'arc (Rühlmann 1885 :472-474, Kurrer 2008, Bénard 2009). Ce faisant, ils mirent à jour l'un des principes de base de la statique graphique, que Karl Culmann intégra à sa théorisation générale.

Dans un autre domaine, celui des ponts suspendus, les ingénieurs français commencèrent à chercher la méthode de calcul à appliquer, lorsque, dans ces mêmes années 1820-1830, la construction des ponts à suspension métallique se développa en Europe $^{41}$, Navier au premier chef (Cannone et Friedlander 2003), mais aussi Seguin 
(Cotte 1993) et Poncelet (Chatzis 1998 et 2004). Polytechnicien, mécanicien, expérimentateur et grand géomètre, Jean-Victor Poncelet reprit les polygones des forces et funiculaire développés par Charles Camus (Chatzis 2004) ${ }^{42}$. Il proposa une solution graphique habile et simple pour déterminer les forces subies par les cordes porteuses: la dualité du polygone des forces et funiculaire. De surcroît, son apport fondamental en géométrie projective a été intégré dans l'élaboration de la statique graphique $^{43}$.

\section{Création d'un système complexe : la statique graphique} est proposée dès le premier chapitre, où il décrit la composition et l'équilibrage des forces. Puis, il étudie les règles de colinéarité et de réciprocité entre le polygone des forces et le polygone funiculaire, en incluant les courbes du second degré ainsi que les différentes positions de leurs points focaux. Le troisième chapitre se focalise sur les effets des moments et des forces sur la surface; le quatrième traite des forces dans l'espace et le cinquième des forces parallèles ainsi que leur moment commun. Pour donner un exemple pratique, il étudie l'impact des roues des wagons de chemin de fer sur le tablier d'un pont. Il s'occupe ensuite, en chapitre 6 , du centre de gravité des forces, des lignes droites, des lignes brisées, des arcs et des lignes générales, puis du centre de gravité des formes et du centre de gravité des corps. Le septième chapitre s'intéresse aux moments d'inertie, le huitième à la construction de l'ellipse et l'ellipsoïde d'inertie et au noyau de la section transversale.

les deux parties qui suivent, il traite des poutres simplement supportées (partie 3) et des poutres continues (partie 4). Consacrée aux structures en treillis, la cinquième partie en présente d'abord la théorie, puis définit la poutre en treillis, le cours des efforts internes exercés sur la poutre en treillis, les poutres en treillis de von Pauli ${ }^{45}$ et la structure du toit. La sixième partie s'intéresse aux arcs: calcul des voûtes, conception graphique de la ligne de pression dans divers types d'arcs, et dans les structures suspendues ${ }^{46}$; les échafaudages et leur stabilité; les structures de renforcement. Les questions économiques, coûts de construction; économies obtenues par l'optimisation graphique de sa forme; le calcul du prix de la structure préfabriquée et la comparaison de coûts entre les différents systèmes de construction occupent la septième partie. Enfin l'ouvrage s'achève, en huitième partie, par l'étude des murs de soutènement. 

Culmann a imaginé l'application systématique des méthodes graphiques dans la résolution de divers problèmes d'ingénierie (Timoshenko 1953). Il présente en outre un certain nombre de solutions graphiques de base. Il décrit comment calculer graphiquement la réaction des poutres, le cours du moment de flexion, comment trouver ses maximums en dessous d'un système de corps mobile. Il cherche le moyen de calculer le centre de gravité d'une section transversale et des moments d'inertie, les principaux axes de la coupe transversale, l'ellipse d'inertie et le noyau de la section transversale. Concernant la poutre courbée, il parvient à calculer graphiquement la contrainte en tout point. Pour les constructions en treillis, il introduit une méthode graphique en coupe transversale et de contact pour déterminer les forces inconnues dans les cannes. Enfin, il conçoit une méthode graphique pour calculer les voûtes, incluant la position de la ligne de pression et les solutions des pressions de la terre.

La réception de l'ouvrage fut à la hauteur des multiples réponses apportées : la statique graphique s'est diffusée dans les pratiques d'ingénierie puis dans l'enseignement en tant que système complet. De ce point de vue, il est intéressant de comparer la préface rédigée par Culmann pour la première édition et celles qu'il rédige, dix ans après, pour l'édition de 1875, où il répond aux arguments des spécialistes et savants que la publication originelle avait impressionnés. Culmann cite, entre autres, les travaux de Maxwell (Maxwell 1864a et b, 1872) ${ }^{47}$ sur les diagrammes de forces et sur le calcul de l'équilibre et de la rigidité des cadres, ainsi que ceux de Luigi Cremona ${ }^{48}$, qui, quelques années plus tard, proposait les mêmes solutions (Cremona 1872 et 1874$)^{49}$. Les modèles de force résultant furent ensuite construits tout au long de l'utilisation de la statique graphique, sous le nom d'épure de Cremona, en Europe continentale, alors que dans les pays anglo-saxons, ils étaient attribués à Maxwell.

\section{BIBLIOGRAPHIE}

AITA Danila, « Between Geometry and Mechanics: a Re-examination of the Principles of Stereotomy from a Statical Point of View », Proceedings of the First International Congress on Construction History, Madrid, 2003, p.161-170

AVILER Augustin-Charles d', Cours d'architecture qui comprend les ordres de Vignole, Paris, Nicolas Langlois, 1691

BENVENUTO Edoardo, An Introduction to the History of Structural Mechanics, Part I: Statics and Resistance of Solids; Part II: Vaulted Structures and Elastic Systems, New York, Springer Science, 1991

BÉNARD Dominique, «Gabriel Lamé et le polygone funiculaire », Bulletin de la Sabix, n44, 2009, p. 113-118 ; DOI : https://doi.org/10.4000/sabix.645

CAIS Stanislav, Statika stavebních konstrukcí-Dějiny stavební mechaniky, Praha, ES ČVUT, 1991

CAMUS Charles Étienne Louis, Cours de mathématique, Paris, Ballard, 1749-1752 
CANNONE Marco, FRIEDLANDER Susane, « Navier: Blow-up and Collapse », Notices of the AMS, Vol. $50, \mathrm{n}^{\circ} 1,2003, \mathrm{p} .7-13$

CHARLTON Thomas Malcom, A History of Theory of Structures in the Nineteenth Century, Cambridge, Cambridge University Press, 1982

CHATZIS Konstantinos, «Jean-Victor Poncelet (1788-1867) ou le Newton de la mécanique appliquée », Bulletin de la Sabix, n¹9, 1998, p.69-97 ; DOI : https://doi.org/10.4000/sabix.857

CHATZIS Konstantinos, « La réception de la statique graphique en France durant le dernier tiers du XIX siècle ", Revue d'histoire des mathématiques, n¹0, 2004, p.7-43

CHOBOT Karel, BENDA Jiři, HÁJEK Văclav, NOVOTNÁ Hájek, Statika stavebních konstrukcí, I. a II, Praha, SNTL, 1982

COTTE Michel, «L'approche mathématique du pont suspendu chez Marc Seguin, 1822-1826 », Revue d'histoire des sciences, t.46, n²-3, 1993, p.233-257 ; DOI : https://doi.org/10.3406/rhs. 1993.4274

CREMONA Luigi, Le figure reciproche nella statica grafica, Milan, Giuseppe Bernardoni, 1872

CREMONA Luigi, Elementi di Calcolo grafico, Torino, Instituti tecnici, 1874

CULMANN Karl, Die Graphische Statik, Zürich, Meyer \& Zeller, 1866

CURL James Stevens, Dictionary of Architecture, Oxford, Oxford University Press, 1999

FAVARO Antonio, DE LUNGO Isidoro, GOVI Gilberto, SCHIAPARELLI Giovanni Virginio (ed.), Le opere di Galileo Galilei: edizione nazionale sotto gli auspicii di sua maesta il re d'Italia, Firenze, G. Barbera, 1890-1909

FEDOROV Sergej G., « Early prefabricated Iron-ribbed Domes: St. Isaac's Cathedral in St. Petersburg, Russia, 1838-1841», Proceedings of 5th International Congress on Construction History, Chicago, 2015, vol.2, p.61-70 ; URL : https://bautechnikgeschichte.files.wordpress.com/2015/07/ fedorov.pdf

FRÉZIER Amédée François, La théorie et la pratique de la coupe des bois et des bois, pour la construction des voies et autres parties des bâtiments civils et militaires, ou Traité de stéréotomie à l'usage de l'architecture, Paris, Doulsseker \& Jombert, 1737-39

HEYMAN Jacques, The Stone Skeleton. Structural Engineering of Masonry Architecture, Cambridge, Cambridge University Press, 1995

HEYMAN Jacques, Structural Analysis. A Historical Approach, Cambridge, Cambridge University Press, (1998) 2003

HOOK Robert, Description of Helioscopes and some other Instruments, London, John \& Martyn Printer to the Royal Society, 1675-76

KURRER Karl Eugen, The History of the Theory of Structures, Berlin, Ernst et Sohn, 2008

LAPPARENT Albert de, « Notice sur Gabriel Lamé », Livre du centenaire de l'École polytechnique, Paris, Gauthier-Villars, t.1, 1897, p.120ss (http://www.annales.org/archives/x/lame.html)

LAMÉ Gabriel, CLAPEYRON Benoît, « Mémoire sur la stabilité des voûtes », Annales des Mines, Treuttel et Wurtz, Paris 1823, p.789-836 ; URL : http://annales.ensmp.fr/articles/ 1823/402-425.pdf

LA HIRE Philippe de, Nouvelle méthode en géométrie pour les sections des superficies coniques et cylindriques, Paris, Moette, 1673 
LA HIRE Philippe de, Traité de Mécanique ou l'on explique tout ce qui est nécessaire dans la pratique des arts, \& les propriétés des corps pesants lesquelles ont un plus grand usage dans la physique, Paris, J. Anisson, 1695 ; Deuxième édition à titre posthume : Paris, Compagnie des Libraires, 1729 LA HIRE Philippe de, «Sur la construction des voûtes dans les édifices », 1712, Mémoires de l'Académie royale des sciences, vol.69, Paris, 1731

LANGE Marc, « A Tale of Two Vectors », Dialectica, vol.63, n, 2009, p.397-431; DOI : https:// doi.org/10.1111/j.1746-8361.2009.01207.x

LOPEZ Giovanni Morello, « Poleni's Manuscripts about the Dome of Saint Peter's ", Proceedings of the Second International Congress on Construction History, Cambridge, 2006, p.1957-1979

MONTFERRAND Auguste Ricard de, Plans de l'Église de Saint-Isaac, St. Petersbourg, 1828 MAXWELL James Clerck, « On reciprocal figures and diagrams of forces », Philosophical Magazine, vol.27, 1864, p.250-261

MAXWELL James Clerck, " On the calculation of the equilibrium and stiffness of frames ", Philosophical Magazine, vol.27, 1864, p.294-299

MAXWELL James Clerck, « On reciprocal figures, frames and diagrams of forces », Transactions of the Royal Society of Edinburgh, vol.26, 1872, p.1-40, planches 1-3

NEWTON Isaac, Philosophia Naturalis Principia Mathematica, Jussu Societatis regiae (Londini), 1687

PICON Antoine, L'art de l'ingénieur. Un art limite, Paris, Éditions du Centre Pompidou - Le Moniteur, 1997

PISANO Raffaele, CAPECCHI Danilo, Tartaglia's Science of Weights and Mechanics in the Sixteenth Century, New York, Springer, 2016

PONCELET Jean-Victor, Cours de mécanique industrielle, t.2, Paris, Clouet, 1828

POSPÍŠIL Martin, Grafická statika (historické kořeny moderní metody výuky statiky pro architekty), Habilitační práce, ČVUT v Praze, 2016

POSPÍŠIL Martin, Kamenořez (odraz stavební mechaniky v konstrukci historických oblouků)/Stereotomy (Reflections of Structural Mechanics in Historical Arches Construction), Thesis, ČVUT Praha, 2007

ROBERTSON Edmund, O'CONNOR John, The MacTutor History of Mathematics Archive, University of St Andrews, 2003 ; URL : https://mathshistory.st-andrews.ac.uk/Biographies/La_Hire/

RÜHLMANN Moritz, Vorträge über geschichte der technischen Mechanik und theoretischen Maschinenlehre, Leipzig, Baumgärtner's Buchhandlung, 1885

TIMOSHENKO Stephen, History of Strength of Materials. With a brief account of the history of theory of elasticity and theory of structures. New York, McGraw-Hill, 1953

VARIGNON Pierre, Nouvelle mécanique ou Statique, Paris, Claude Jombert, 1687

VENTURI Giovanni Battista, Essai sur les ouvrages physico-mathématiques de Léonard de Vinci, Paris, Duprat, 1797

WHITESIDE Derek Thomas (ed.), The Mathematical Papers of Isaac Newton, Vol. 6, 1684-1691, Cambridge, New York, Cambridge University Press, 1974 


\section{NOTES}

1. Ainsi, la statique graphique a été utilisée pour le calcul de vérification du plus grand bâtiment d'habitation au monde, Burj Khalifa, haut de plus de $800 \mathrm{~m}$ de haut. Ce bâtiment a été inauguré en 2010. Source : cf. entretien avec l'expert en statique en chef de ce bâtiment William Frazier Baker /Skidmore, Owings \& Merrill, LLP/ dans le documentaire MegaStructures. World's Tallest Skyscraper, National Geographic Channels, 2012.

2. Ingénieur allemand formé à l'École polytechnique de Karlsruhe, Karl Culmann (1821-1881) réalisa des projets de ponts pour les chemins de fer en Bavière. Entre 1849 et 1851, il effectua des voyages d'études en Grande-Bretagne et aux États-Unis. À la suite de quoi, il exerça comme professeur de génie civil à l'ETH (Eidgenössische Technische Hochschule) de Zurich, de 1855 à sa mort. Son ouvrage principal, Die Graphische Statik, a commencé à être publié en 1864.

3. Par exemple, le calcul du toit de la grande salle de cinéma de l'Hôtel Thermal à Karlovy Vary, effectué par le professeur Ferdinand Lederer et son équipe, repose sur des calculs de vérification faits à l'aide de la statique graphique et inscrits directement sur les dessins du permis de construire.

4. Par exemple : la statique de corps solide d'Archimède (III ${ }^{\mathrm{e}}$ siècle avant $\mathrm{J} .-\mathrm{C}$.), l'équilibre du moment de Thabit ibn Kurra, également traducteur arabe d'Archimède, (IX ${ }^{\mathrm{e}}$ siècle après J.-C.), ou encore le poids comme force de Jordanus Nemorarius (XIII ${ }^{\mathrm{e}}$ siècle).

5. Tratado de Estatica y Mecanica En Italiano (Codex de Madrid), 1493, Biblioteca Nacional, Madrid, MSS/8937.

6. Notebook of Leonardo da Vinci (Codex Arundel), 1478-1518, British Museum, London, MS 263. Lors de l'exposition proposé au public entre février au septembre 2016, le Science Museum de Londres s'est appesanti sur le travail et les inventions de l'ingénieur en mécanique.

7. Codex de Madrid, fol. 63r až fol. 67r.

8. Codex de Madrid, fol. 91r ad.

9. Codex de Madrid, fol. 88 recto \& verso.

10. Codex de Madrid, fol. 139v.

11. Codex Arundel, fol. 1v (sign.263).

12. Institut de France, Paris, 4E N.S. 8993.

13. Simon Stevinus (1548-1620), mathématicien flamand, contribua à la standardisation du système décimal ( $L a$ Thiende, La Disme, tous deux parus en 1585) et à la réfutation de l'argument d'Aristote selon lequel les corps plus lourds tombent plus rapidement que les corps plus légers (rapport de 1586). Dans De Beghinselen der Weeghconst (1586), il forme un triangle de forces.

14. De Beghinselen der weeghconst, beschreven duer Simon Stevin van Brugghe, Druckerye van C. Plantijn, Leyden 1586 (BnF V-7241(1)). Cette preuve est également connue sous le nom « d'épitaphe de Stevin ».

15. À l'époque, cette expérience était aussi une preuve importante et très démonstrative du manque de mobilité perpétuelle (perpetuum mobile).

16. Galileo Galilei, plus connu sous le nom de Galilée (1564-1642), physicien, mathématicien et astronome italien, a contribué de manière décisive à l'étude du mouvement du corps, de la résistance des matériaux, de l'astronomie et de la méthodologie scientifique.

17. Discorsi e dimostrazioni matematiche intorno a due nuove scienze attenenti alla mecanica $e i$ movimenti locali, del signor Galileo Galilei. Leida : Appresso gli Elsevirii, 1638 (BnF 4-S-3586).

18. Il s'agit là d'un véritable tournant que Galilée a fait inscrire dans les Discorsi après 1639 (FAVARO et al. 1890-1909, partie VIII, BnF4-Z-557 (1)); dans la première édition de 1638, cette note n'est pas présente (BnF 4-S-3586).

19. Isaac Newton (selon le calendrier julien 1642, selon le grégorien 1643-1727), physicien et mathématicien anglais, contributions essentielles en mécanique (lois du mouvement), en optique (composition de la lumière blanche), en mathématiques (nombre infiniment petit), etc. 
20. Newton 1687, BnF ark:/12148/bpt6k3363w.

21. Pierre Varignon (1654-1722), mathématicien français, connu pour sa description de l'écoulement des fluides par calcul différentiel (Encyclopedia Britannica et www.bibmath.net).

22. Varignon 1687, ETH Zürich Bibliothek : Rar4673 :1.

23. Philippe de la Hire (1640-1718), mathématicien français, géomètre, astronome, architecte (Robertson, O'Connor 2003 : « Philippe de la Hire »).

24. La Hire 1673 (BnF V-6274).

25. Robert Hooke recommande l'ouvrage de La Hire à Isaac Newton dans sa lettre du 24 novembre 1679 (Whiteside 1974 : 268-272).

26. "The solution lays in La Hire's invention of the force polygon and the corresponding funicular polygon for the arch (which is nothing more than the line of thrust, or the shape of the inverted hanging chain, although La Hire did not use the terms) " ("la solution réside dans l'invention par de La Hire du polygone de force et du polygone funiculaire correspondant pour l'arc, qui n'est autre que la ligne de poussée ou la forme de la chaine suspendue inversée, bien que la Hire n'ait pas utilisé ces termes ») (Heyman $1998: 81$ ).

27. La Hire s'appuie sur le fait que l'arc serait altéré dans la partie située entre le talon et le dernier voussoir, au niveau du joint entre les voussoirs, approximativement à $45^{\circ}$ de la verticale. La nature de l'altération représente la rotation virtuelle du quart supérieur de la voûte par rapport au quart inférieur, autour du point hypothétique situé sur la face intérieure de la voûte. Cependant, il ne spécifie aucune règle pour trouver une position plus précise de ce point, ni les conditions dans lesquelles l'arc doit atteindre l'état limite de l'altération.

28. En fait, jusqu'à la solution proposée par Charles Coulomb en 1773-1775 (Heyman 1998 :82-83). 29. Charles Étienne Louis Camus (1699-1768), architecte du roi, mathématicien et géomètre, membre de l'équipe de mesure du méridien (1736) et l'un des auteurs de la Carte de la France, dirigea l'Académie royale des sciences de 1750 et 1761 (Robertson, O'Connor 2013 : « CharlesEtienne-Louis Camus »); Camus 1749 (BnF V-18559).

30. Près d'un siècle plus tard, Jean-Victor Poncelet présenta une solution presque identique dans le cadre des ponts à haubans (Poncelet $1828: 74$; Chatzis $2004: 12-14$ ).

31. Giovanni Poleni (1683-1761), physicien italien, professeur d'astronomie et de météorologie à l'Université de Padoue. Il mena des relevés météorologiques précis pendant quarante ans qui servirent de base à d'autres mesures météorologiques. Il est l'auteur d'un certain nombre de traités sur les instruments météorologiques. En 1748, il publia la Memorie storiche della gran Cupola del Tempio Vaticano, Padua (Robertson, O'Connor 2003 : « Giovanni Poleni »).

32. Parere di tre mattematici sopra i danni che si sono trovati nella cupola di S. Pietro sul fine dell'anno 1742, Rome, 1743.

33. Il s'agirait de la première évaluation structurelle connue à ce jour (Straub 1952, p.123 ; Benvenuto 1991, p.351; Heyman 1998, p.86ss.). Quoiqu'il en soit, l'évaluation a été faite de manière très moderne, comme on peut le constater à l'application du principe des états limites (la formation de fissures dans le dôme).

34. Robert Hooke (1635-1703) avait découvert que la forme idéale d'une voûte est donnée par une chaînette de suspension que l'on renverse. Il l'exprima dans son ouvrage sur les hélioscopes, sous la forme d'anagrammes: l'anagramme «ceiiinosssttuu» = "ut tensio, sic vis» signifiant: "la déformation est proportionnelle à la tension », ce qui est la description du postulat de l'élasticité du matériau ; l'anagramme : « abcccddeeeefggiiiiiiiillillmmmmnnnnooprrssstttttttttuuuuuuux » = «Ut pendet continuum flexile, sic stabit contiguum rigidum inversum », signifiant : «Comme pend la ligne flexible inversée, ainsi se tiendra l'arche rigide » (Hook 1675-76). C'est la plus ancienne trace écrite connue de la recherche de la forme idéale de l'arc (Heyman 1995 :7 ; Heyman 1998 : 79). 
35. "Stéréotomie: science qui enseigne la coupe des solides, comme dans les profils d'Architecture des murs, voûtes, \& autres solides coupés. Ce mot vient du Grec Stereos, solide, \& Tomè, section » (Aviler $1691: 829$ ).

36. Danila Aita cite la description de la stéréotomie par Deschalles comme un exemple de l'enlèvement de la pierre en une intersection centrale (Aita $2003: 164$ ). Mais ce procédé est présent chez Vitruve et Villard de Honnecourt (Pospísil 2007).

37. Dans le milieu tchèque, par exemple, la stéréotomie et la mécanique des structures étaient également liées. Au XVIII ${ }^{\mathrm{e}}$ siècle, František Josef Gerstner fut actif dans les deux disciplines (Gerstner F.J., Einleitung in die statische Baukunst, Prague, 1789), de même, au XIX ${ }^{\mathrm{e}}$ siècle, que Josef Šlín (Šolín J., Stéréotomie, Prague, 1899) et František Klokner, qui enseigna à la fois la mécanique des structures et la stéréotomie, et Zdeněk Bažant, important ingénieur tchèque en structures, nommé professeur de stéréotomie à Prague en 1917 (Pospísil 2007).

38. Augustin A. Danyzy ou Danisi (1698-1777). Il fut professeur de mathématiques et d'hydrographie à l'Académie des sciences de Montpellier de 1740 à 1777.

39. Diplômés de l'École polytechnique et de l'École des Mines de Paris, Benoît Clapeyron (1799-1864) et Gabriel Lamé (1795-1870), travaillèrent de concert une grande partie de leur carrière. Leurs diplômes en poche, en 1820, ils partirent enseigner les mathématiques pures et appliquées à l'École des Travaux publics de Saint-Pétersbourg. De retour en France, en 1830, B. Clapeyron devint l'un des promoteurs du chemin de fer en France (Paris-St-Germain, ligne de Versailles, chemin de fer du Nord, lignes du Midi ; il s'occupa de la reconstruction du pont d'Asnières, après 1848, et devint l'un des théoriciens des ponts suspendus et des voûtes en maçonnerie, les machines à vapeur. Il fut élu à l'Académie des sciences en 1858. Quant à Gabriel Lamé, il occupa la chaire de physique à l'École polytechnique. Élu à l'Académie des sciences en 1843, il devint professeur à la Sorbonne en 1850, sur la chaire de Calcul des probabilités, qu'il transforma en chaire de Physique mathématique (http://www.annales.org/archives/x/ clapeyron.html; http://www.annales.org/archives/x/lame.html).

40. La publication dans les Annales des Mines comporte le rapport de Prony. Le mémoire fut également publié en Russie en 1826, dans le Journal des voies de communication. La construction du dôme fut réalisée en 1838-1841 sous la direction de Montferrand et Baird (Montferrand $1828: 3$, tab.7 ; Fedorov 2015).

41. Le premier pont moderne à suspension métallique fut construit en 1801 sur la Jacob's Creek en Pennsylvanie par James Finley. Le premier pont européen (Union Bridge) fut construit sur la rivière Tweed entre l'Angleterre et l'Écosse par Samuel Brown en 1820 ; dans les pays tchèques, le premier pont suspendu à chaînes fut le pont du comte Franz Magniss à Straznice en Moravie, réalisé par Bedřich Schnirch en 1823-24; en 1824-1825, Marc Seguin fit construire le pont suspendu qu'il projetait depuis 1822, sur le Rhône, à Tournon-Tain (Cotte 1993).

42. Jean-Victor Poncelet (1788-1867). Mathématicien et physicien français (mécanique), il fut l'élève de Gaspard Monge à l'École polytechnique de Paris, puis à Metz. En 1812, il se rend avec Napoléon en Russie où il est capturé et ne rentre en France qu'en 1814. En 1822, il publie son Traité des propriétés projectives des figures qui renouvelle le domaine de la géométrie pure ; en 1824, il présente sa roue hydraulique à aubes courbes, la « roue Poncelet » qui renouvelle l'usage de la roue hydraulique dans l'industrie. Professeur de mécanique à l'école d'artillerie de Metz en 1825, il entre à l'Académie des sciences en 1834. Devenu professeur à la Sorbonne, il crée le cours de mécanique appliquée. Nommé général en 1848, il devient directeur de l'École polytechnique. Dans le domaine de la mécanique, outre la roue Poncelet, il a travaillé sur la résistance des matériaux et proposé un diagramme de test de traction. En géométrie, il est considéré comme le fondateur de la géométrie projective moderne (Timoshenko 1953 : 87-88).

43. Le Traité des propriétés projectives des figures est considéré comme le travail de base de la géométrie projective moderne. (Robertson, O'Connor 2003 : «Jean-Victor Poncelet »). 
44. La publication de l'ouvrage s'est faite sur deux ans : les parties 1 (Das Graphische Rechnen) et 2 (Die Graphische Statik) ont été publiées en 1864 (Maurer, p.151) et les parties 3 (Der Balken), 4 (Der continuirliche Balken), 5 (Das Fachwerk), 6 (Bogen), 7 (Der Werth der Constructionen) et 8 (Theory der Stütz- und Futtermauern) ainsi que les deux premières parties, une préface, une correction et un complément, dans son ensemble, ont été publiées en 1866 sous le titre Die Graphische Statik von K. Culmann (Zurich, Verlag von Meyer \& Zeller, 1866).

45. Friedrich August von Pauli (1802-1883), ingénieur civil allemand, concepteur des ponts de chemin de fer en métal des chemins de fer bavarois, a développé une forme typiquement lenticulaire des treillis.

46. En 1851 et 1852, Culmann a publié la revue Allgemeine Bauzeitung deux articles d'une soixantaine de pages (vol.16, p.69-129; vol.17, p.163-222) sur les ponts anglais et américains. Culmann y évoque les systèmes de conception de ponts au Royaume-Uni et aux États-Unis, ainsi que la manière dont ils ont été calculés et testés. (Timoshenko 1953 :191-194).

47. James Clerk Maxwell (1831-1879), mathématicien et physicien écossais. On lui doit une description mathématique générale du champ électromagnétique (équations de Maxwell), la théorie de l'additivité des couleurs, une théorie cinétique et thermodynamique (division de Maxwell-Boltzman).

48. Luigi Cremona (1830-1903), mathématicien italien, professeur de géométrie à l'Université de Bologne, réformateur de l'enseignement des mathématiques et de la géométrie au XIX ${ }^{\mathrm{e}}$ siècle en Italie, créateur des motifs de Cremona en statique graphique. (Robertson, O'Connor 2003: «Luigi Cremona »).

49. Cremona était probablement au courant des articles de Maxwell (Charlton 1982 : 60-62).

\section{RÉSUMÉS}

Le début de la statique graphique est généralement associé aux travaux de l'ingénieur allemand Karl Culmann (1821-1881), avec son ouvrage Die Graphische Statik, publiés en huit volumes à Zurich en 1864-1866. Pourtant Culmann le souligne dans la préface de son œuvre: il a pris en compte les études conduites par les savants et ingénieurs qui ont ouvert le champ de la statique graphique, notamment Jean-Victor Poncelet (1788-1867), Barthélemy Cousinery (1790-1851), Jakob Steiner (1796-1863). Et, comme il le mentionne également, les origines de la statique graphique remontent bien avant. Dans cet article, nous présentons les trois fondements de la statique graphique: i) la composition et la décomposition des forces avec le polygone funiculaire ; ii) les stimuli venus des pratiques de construction; iii) la dualité forme-force.

The beginning of graphic statics is generally associated with the work of the German engineer Karl Culmann (1821-1881), and his Die Graphische Statik, published in eight volumes in Zurich in 1864-1866. However, Culmann emphasises in his preface that he took into account the studies carried out by the scientists and engineers who had opened up the field of graphic statics, notably Jean-Victor Poncelet (1788-1867), Barthélemy Cousinery (1790-1851), Jakob Steiner (1796-1863). And, as he also mentions, the origins of graphic statics go back much further. In this paper we present the three foundations of the graphic statics: i) the composition and decomposition of forces with the funicular polygon; ii) the stimuli from building practices; iii) the duality between the shape of the structure and the internal force diagram. 


\section{INDEX}

Mots-clés : histoire des techniques, histoire des sciences, mécanique structurale, construction, ingénieur, génie civil, architecture, académie royale des sciences

Keywords : history of technology, history of science, structural mechanics, building, engineer, civil engineering

\section{AUTEUR}

\section{MARTIN POSPÍŠIL}

Professeur agrégé au Département des structures porteuses de la Faculté d'architecture de l'Université polytechnique de Prague, Martin Pospíšil a débuté sa carrière professionnelle en tant que tailleur de pierre. Après ses études de maîtrise en génie civil (Université technique de Brünn), il est devenu ingénieur-architecte puis superviseur d'État de construction de bâtiments (conception et supervision de plans de rénovation de bâtiments protégés). En 2008, il a terminé son doctorat en ingénierie architecturale et structurelle (Université polytechnique de Prague) et en 2019, il a obtenu son doctorat d'histoire des techniques, en co-diplomation à l'Université Paris 1 Panthéon Sorbonne et à l'Université polytechnique de Prague. Ses recherches portent principalement sur les structures et technologies de bâtiments historiques et sur les nouvelles façons d'enseigner la mécanique des structures aux architectes en se basant sur l'explication et la pratique des technologies historiques. 